\title{
ISOMORPHIC INDUCED MODULES AND DYNKIN DIAGRAM AUTOMORPHISMS OF SEMISIMPLE LIE ALGEBRAS
}

\author{
JÉRÉMIE GUILHOT AND CÉDRIC LECOUVEY
}

\begin{abstract}
Consider a simple Lie algebra $\mathfrak{g}$ and $\overline{\mathfrak{g}} \subset \mathfrak{g}$ a Levi subalgebra. Two irreducible $\overline{\mathfrak{g}}$ modules yield isomorphic inductions to $\mathfrak{g}$ when their highest weights coincide up to conjugation by an element of the Weyl group $W$ of $\mathfrak{g}$ which is also a Dynkin diagram automorphism of $\overline{\mathfrak{g}}$. In this paper we study the converse problem: given two irreducible $\overline{\mathfrak{g}}$-modules of highest weight $\mu$ and $\nu$ whose inductions to $\mathfrak{g}$ are isomorphic, can we conclude that $\mu$ and $\nu$ are conjugate under the action of an element of $W$ which is also a Dynkin diagram automorphism of $\overline{\mathfrak{g}}$ ? We conjecture this is true in general. We prove this conjecture in type $A$ and, for the other root systems, in various situations providing $\mu$ and $\nu$ satisfy additional hypotheses. Our result can be interpreted as an analogue for branching coefficient of the main result of 6] on tensor product multiplicities.
\end{abstract}

\section{INTRODUCTION}

Let $\mathfrak{g}$ be a simple Lie algebra over $\mathbb{C}$ and $\overline{\mathfrak{g}}$ a Levi subalgebra. Let $\mu$ and $\nu$ be two dominant integral weights for $\overline{\mathfrak{g}}$. Denote by $\bar{V}(\mu)$ and $\bar{V}(\nu)$ the associated highest weight $\overline{\mathfrak{g}}$-modules. Let $\bar{V}(\mu) \uparrow \mathfrak{g}$ and $\bar{V}(\nu) \uparrow \overline{\mathfrak{g}}$ be the $\mathfrak{g}$-modules obtained by induction from $\overline{\mathfrak{g}}$. When $\mu$ and $\nu$ are conjugate by an element of the Weyl group $W$ of $\mathfrak{g}$ which is also a Dynkin diagram automorphism of $\overline{\mathfrak{g}}$, the modules $\bar{V}(\mu) \uparrow \mathfrak{g}$ and $\bar{V}(\nu) \uparrow \frac{\mathfrak{g}}{\mathfrak{g}}$ are isomorphic; see Proposition 4.4, In this paper, we address the following question: assume $\bar{V}(\mu) \uparrow \frac{\mathfrak{g}}{\mathfrak{g}}$ and $\bar{V}(\nu) \uparrow \mathfrak{g}$ are isomorphic, can we conclude that $\mu$ and $\nu$ are conjugate by an element of the Weyl group $W$ of $\mathfrak{g}$ which is also a Dynkin diagram automorphism of $\overline{\mathfrak{g}}$ ?

It is interesting to reformulate this problem in terms of the (infinite) matrix $M=\left(m_{\mu}^{\lambda}\right)$ with columns and rows labelled respectively by the dominant weights $\lambda$ of $\mathfrak{g}$ and by the dominant weights $\mu$ of $\overline{\mathfrak{g}}$. Here $m_{\mu}^{\lambda}$ denotes the branching coefficient corresponding to the multiplicity of the irreducible highest weight $\mathfrak{g}$-module $V(\lambda)$ in $\bar{V}(\mu) \uparrow \overline{\mathfrak{g}}$ (or equivalently the multiplicity of $\bar{V}(\mu)$ in the restriction of $V(\lambda)$ to $\overline{\mathfrak{g}})$. We then ask if two rows of the matrix $M$ can be equal. Note that two distinct columns of $M$ labelled by $\lambda$ and $\mu$ of $M$ cannot coincide since this would imply $V(\lambda) \simeq V(\mu)$. Indeed both modules would then have the same weight decomposition and therefore the same characters.

The matrix $M$ contains inner multiplicities associated to simple $\mathfrak{g}$-modules. We can also address a similar question for the outer (tensor product) multiplicities. The corresponding matrix, say $C$, has columns and rows labelled by dominant weights of $\mathfrak{g}$ and $k$-tuples $\left(\mu^{(1)}, \ldots, \mu^{(k)}\right)$ of such dominant weights. The coefficients $c_{\mu^{(1)}, \ldots, \mu^{(k)}}^{\lambda}$ is then the multiplicity of $V(\lambda)$ in $V\left(\mu^{(1)}\right) \otimes \cdots \otimes V\left(\mu^{(k)}\right)$. It was proved by Rajan in [6] (see also [7] for a shorter proof and an extension to the case of Kac-Moody algebras) that two rows of $C$ are equal if and only if the associated $k$-tuples of dominant weights coincide up to permutation. It is also easy to see that if the columns of $C$ labelled by $\lambda$ and $\kappa$ coincide, then $\lambda=\kappa\left(\right.$ take $\left(\mu^{(1)}, \ldots, \mu^{(k)}\right)=(\lambda, 0, \ldots, 0)$ and $\left.\left(\mu^{(1)}, \ldots, \mu^{(k)}\right)=(\kappa, 0, \ldots, 0)\right)$.

Finally, one can also consider the decomposition matrix $D$ associated to the modular representation theory of the symmetric group in characteristic $p$. Its columns and rows are indexed by $p$-restricted partitions and partitions of $n$, respectively. The study of possible identical rows and 
columns was considered by Wildon in [8]: the columns of $D$ are distinct and its rows can only coincide in characteristic 2 when the underlying partitions are conjugate.

In the present paper, we prove that two rows of the matrix $M$ corresponding to weights conjugate by an element of the Weyl group $W$ of $\mathfrak{g}$ which is also a Dynkin diagram automorphism of $\overline{\mathfrak{g}}$ coincide. We conjecture that the converse is true and prove this conjecture in various cases (see Theorem 7.4). We believe that the study of the matrix $M$ is more complicated than that of the matrix $C$ for two main reasons. First, there could exist infinitely many nonzero coefficients in a row of $M$ (this is not the case for $C$ ). Second, the possible transformations relating the labels corresponding to identical rows in $M$ are more complicated than in the case of the matrix $C$ (where they simply correspond to permutations of the $k$-tuples of dominant weights).

The paper is organised as follows. Section 2 is devoted to some classical background on representation theory of Lie algebras. In Section 3, we study the relationships between the roots and the weights of $\mathfrak{g}$ and $\overline{\mathfrak{g}}$. In Section 4 , we formulate our conjecture in terms of equality of distinguish functions in the character ring of $\mathfrak{g}$. This allows us in Section 5 to prove our conjecture when $\mu$ and $\nu$ are far enough from the walls of the Weyl chamber in which they appear. In Section 6 , we prove the conjecture when $\mu+2 \bar{\rho}$ or $\nu+2 \bar{\rho}$ (where $\bar{\rho}$ denotes the half sum of positive roots of $\overline{\mathfrak{g}}$ ) is dominant for $\mathfrak{g}$. Finally, in Section 7 , we prove the conjecture in the case $\mathfrak{g}=\mathfrak{g l}_{n}$ by using the main result of Rajan [6]. This also permits to establish it when $\mathfrak{g}$ is a classical Lie algebra of type $B_{n}, C_{n}$ or $D_{n}$ and $\overline{\mathfrak{g}}=\mathfrak{g l}_{n}$.

\section{BaCKGRound on Lie ALGEBRAS}

Let $\mathfrak{g}$ be a simple Lie algebra over $\mathbb{C}$ with triangular decomposition

$$
\mathfrak{g}=\bigoplus_{\alpha \in R_{+}} \mathfrak{g}_{\alpha} \oplus \mathfrak{h} \oplus \bigoplus_{\alpha \in R_{+}} \mathfrak{g}_{-\alpha}
$$

so that $\mathfrak{h}$ is the Cartan subalgebra of $\mathfrak{g}$ and $R_{+}$its set of positive roots. The root system $R=$ $R_{+} \sqcup\left(-R_{+}\right)$of $\mathfrak{g}$ is realised in a real Euclidean space $E$ with inner product $(\cdot, \cdot)$. For any $\alpha \in R$, we write $\alpha^{\vee}=\frac{2 \alpha}{(\alpha, \alpha)}$ for its coroot. Let $S \subset R_{+}$be the subset of simple roots. The set $P$ of integral weights for $\mathfrak{g}$ satisfies $\left(\beta, \alpha^{\vee}\right) \in \mathbb{Z}$ for any $\beta \in P$ and $\alpha \in R$. We write $P_{+}=\left\{\beta \in P \mid\left(\beta, \alpha^{\vee}\right) \geq 0\right.$ for any $\alpha \in S\}$ for the cone of dominant weights of $\mathfrak{g}$. Let $W$ be the Weyl group of $\mathfrak{g}$ generated by the reflections $s_{\alpha}$ with $\alpha \in R_{+}$(or equivalently by the simple reflections $s_{\alpha}$ with $\alpha \in S$ ). Set $C=\left\{x \in E \mid\left(x, \alpha^{\vee}\right)>0\right\}$ and $\bar{C}=\left\{x \in E \mid\left(x, \alpha^{\vee}\right) \geq 0\right\}$. For any $w \in W$, we set

$$
C_{w}=w^{-1}(C), \quad \bar{C}_{w}=w^{-1}(\bar{C}) \quad \text { and } \quad P_{+}^{w}=P \cap \bar{C}_{w} .
$$

Each set $w^{-1}(S)$ can be chosen as a set of simple roots for $R$, the corresponding set of positive roots is then $R_{+}^{w}=w^{-1}\left(R_{+}\right)$. Given $w \in W$, we define the dominance order $\leq_{w}$ on $P$ by the following relation: $\gamma \leq_{w} \beta$ if and only if $\beta-\gamma$ decomposes as a sum of roots in $R_{+}^{w}$. When $w=1$, we simply write $\leq$ for the order $\leq_{1}$.

Now consider a subset of simple roots $\bar{S} \subset S$. Write $\bar{R} \subset R$ for the parabolic root system generated by $\bar{S}$ and $\bar{R}_{+}=\bar{R} \cap R_{+}$the corresponding set of positive roots. Let $\overline{\mathfrak{g}} \subset \mathfrak{g}$ be the Levi subalgebra of $\mathfrak{g}$ with set of positive roots $\bar{R}_{+}$and triangular decomposition

$$
\overline{\mathfrak{g}}=\bigoplus_{\alpha \in \bar{R}_{+}} \mathfrak{g}_{\alpha} \oplus \mathfrak{h} \oplus \bigoplus_{\alpha \in \bar{R}_{+}} \mathfrak{g}_{-\alpha}
$$

In particular, $\mathfrak{g}$ and $\overline{\mathfrak{g}}$ have the same Cartan subalgebra. The algebras $\mathfrak{g}$ and $\overline{\mathfrak{g}}$ have the same integral weight lattice $P$. The Weyl group $\bar{W}$ of $\overline{\mathfrak{g}}$ is generated by the simple reflections $s_{\alpha}$ with $\alpha \in \bar{S}$. Denote by $\bar{P}_{+} \subset P$ the set of dominant integral weights of $\overline{\mathfrak{g}}$. We shall also need the partial 
order $\preceq$ on $P$ defined by the following relation: $\gamma \preceq \beta$ if and only if $\beta-\gamma$ decomposes as a sum of roots in $\bar{R}_{+}$.

Example 2.1. Consider $\mathfrak{g}=\mathfrak{s p}_{12}$. We have

$$
R_{+}=\left\{\varepsilon_{i}-\varepsilon_{j} \mid 1 \leq i<j \leq 6\right\} \cup\left\{\varepsilon_{i}+\varepsilon_{j} \mid 1<i<j \leq 6\right\} \cup\left\{2 \varepsilon_{i} \mid 1 \leq i \leq 6\right\}
$$

and

$$
P_{+}=\left\{x=\left(x_{1}, \ldots, x_{6}\right) \in \mathbb{Z}^{6} \mid x_{1} \geq \cdots \geq x_{6} \geq 0\right\} .
$$

The Levi subalgebra $\overline{\mathfrak{g}} \subset \mathfrak{g}$ such that

$$
\bar{R}_{+}=\left\{\varepsilon_{1}-\varepsilon_{2}, \varepsilon_{1}-\varepsilon_{3}, \varepsilon_{2}-\varepsilon_{3}\right\} \cup\left\{\varepsilon_{4} \pm \varepsilon_{5}, \varepsilon_{4} \pm \varepsilon_{6}, \varepsilon_{5} \pm \varepsilon_{6}\right\} \cup\left\{2 \varepsilon_{4}, 2 \varepsilon_{5}, 2 \varepsilon_{6}\right\}
$$

is then isomorphic to $\mathfrak{g l}_{3} \oplus \mathfrak{s p}_{6}$.

Given $\lambda \in P_{+}$, we denote by $V(\lambda)$ the finite dimensional irreducible representation of $\mathfrak{g}$ with highest weight $\lambda$. Let $s_{\lambda}$ be the character of $V(\lambda)$. This is an element of the group algebra $\mathbb{Z}[P]$ with basis $\left\{e^{\beta} \mid \beta \in P\right\}$. More precisely

$$
s_{\lambda}=\sum_{\mu \in P} \operatorname{dim} V(\lambda)_{\mu} e^{\mu}
$$

where $V(\lambda)_{\mu}$ is the weight space in $V(\lambda)$ corresponding to $\mu$. Set $\mathbb{G}=\mathbb{Z}^{W}[P]$. We then have $s_{\lambda} \in \mathbb{G}$, that is $s_{\lambda}$ is symmetric under the action of $W$. We also recall the Weyl character formula

$$
s_{\lambda}=\frac{\sum_{w \in W} \varepsilon(w) e^{w(\lambda+\rho)-\rho}}{\prod_{\alpha \in R_{+}}\left(1-e^{-\alpha}\right)}
$$

where $\rho=\frac{1}{2} \sum_{\alpha \in R_{+}} \alpha$. Note that, for any $w \in W$ and $\beta \in P$, we have $s_{w(\beta)}=\varepsilon(w) s_{w \circ \beta}$ where $\circ$ is the dot action of the Weyl group defined by $w \circ \beta=w(\beta+\rho)-\rho$.

Using the restriction of $V(\lambda)$ to $\overline{\mathfrak{g}}$ we define the branching coefficients $m_{\mu}^{\lambda}$ by

$$
s_{\lambda}=\sum_{\mu \in \bar{P}_{+}} m_{\mu}^{\lambda} \bar{s}_{\mu}
$$

where $\bar{s}_{\mu}$ is the character of the irreducible representation $\bar{V}(\mu)$ of $\overline{\mathfrak{g}}$ of highest weight $\mu$. We introduce the partition function $\overline{\mathcal{P}}$ defined by

$$
\prod_{\alpha \in R_{+} \backslash \bar{R}_{+}} \frac{1}{1-e^{\alpha}}=\sum_{\beta \in P} \overline{\mathcal{P}}(\beta) e^{\beta} .
$$

Then, the branching coefficient $m_{\mu}^{\lambda}$ can be computed in term of $\overline{\mathcal{P}}$ using the Weyl character formula (see [3, p. 357]).

Theorem 2.2. Let $\lambda \in P_{+}$and $\mu \in \bar{P}_{+}$. Then

$$
m_{\mu}^{\lambda}=\sum_{w \in W} \varepsilon(w) \overline{\mathcal{P}}(w(\lambda+\rho)-\mu-\rho)
$$

where $\varepsilon$ is the sign representation of $W$. 


\section{Dominant weights of $\overline{\mathfrak{g}}$ And Weyl Chambers}

This section is devoted to study the relationship between the various subsets of roots and weights we have defined. To this end we introduce the following subset which will play an important role in this paper:

$$
U=\left\{u \in W \mid u\left(\bar{R}_{+}\right) \subset R_{+}\right\}
$$

Proposition 3.1. We have

(1)

$$
\begin{aligned}
& \bar{P}_{+}=\bigcup_{u \in U} u^{-1}\left(P_{+}\right) . \\
& \bar{R}_{+}=\bigcap_{u \in U} u^{-1}\left(R_{+}\right) .
\end{aligned}
$$

(3) Each element $w$ in $W$ admits a unique decomposition under the form $w=u \bar{w}$ with $u \in U$ and $\bar{w} \in \bar{W}$.

Proof. We prove 1. Let $\lambda \in P_{+}$and $u \in U$. For all $\alpha \in \bar{R}_{+}$, we have

$$
\left(u^{-1}(\lambda), \alpha^{\vee}\right)=\left(\lambda, u(\alpha)^{\vee}\right) \geq 0
$$

since $\lambda \in P_{+}$and $u(\alpha) \in R_{+}$. It follows that $u^{-1}(\lambda) \in \bar{P}_{+}$and $\bigcup_{u \in U} u^{-1}\left(P_{+}\right) \subset \bar{P}_{+}$.

Next let $\gamma \in \bar{P}_{+}$. There exists $u^{\prime} \in W$ such that $u^{\prime}(\gamma) \in P_{+}$. Let $\alpha \in \bar{R}_{+}$. Then $(\gamma, \alpha)=$ $\left(u^{\prime}(\gamma), u^{\prime}(\alpha)\right) \geq 0$. If the inequality is strict then we have $u^{\prime}(\alpha) \in R_{+}$. We set

$$
\begin{aligned}
R_{>0} & :=\left\{\beta \in R \mid\left(u^{\prime}(\gamma), \beta\right)>0\right\} \subset R_{+}, \\
R_{0} & :=\left\{\beta \in R \mid\left(u^{\prime}(\gamma), \beta\right)=0\right\} \\
R_{0,+} & :=\left\{\beta \in R_{+} \mid\left(u^{\prime}(\gamma), \beta\right)=0\right\}, R_{0,-}=-R_{0,+} .
\end{aligned}
$$

Note that $R_{0}$ is a subroot system of $R$ and that the simple system associated to $R_{0,+}$ consists simply of $R_{0,+} \cap S$. Also, since $u(\gamma) \in P_{+}$, we have $R_{+}=R_{>0} \cup R_{0,+}$. Let $W_{0}=\left\langle s_{\beta} \mid \beta \in R_{0}\right\rangle$. The group $W_{0}$ then acts on $R$ and stabilises both $R_{0}$ and $R_{>0}$. Since all the roots in $R_{0}$ are orthogonal to $u^{\prime}(\gamma)$ we have $v u^{\prime}(\gamma)=u^{\prime}(\gamma) \in P_{+}$for all $v \in W_{0}$. Now let $u$ be the element of minimal length in the coset $W_{0} u^{\prime}$. By the previous argument, we do have $u^{\prime}(\gamma) \in P_{+}$. Let us show that $u \in U$. Let $\alpha \in \bar{R}_{+}$. First if $u^{\prime}(\alpha) \in R_{>0}$, then so does $u(\alpha)$ since $W_{0}$ stabilises $R_{>0}$ and we are done in this case since $u(\alpha) \in R_{>0} \subset R_{+}$. Second, if $u^{\prime}(\alpha) \in R_{0}$, then so does $u(\alpha)$. Let $\delta \in R_{0,+} \cap S$. Since $u$ is of minimal length, we have $\ell\left(s_{\delta} u\right)>\ell(u)$ (here $\ell$ is the length function) and this implies that $u^{-1}(\delta) \in R_{+}$(see for example [4, $\left.\S 1.6\right]$ ). It follows that $u^{-1}(\beta)$ is positive for all $\beta \in R_{0,+}$. Therefore we cannot have $u(\alpha)=-\beta \in R_{0,-}$ with $\beta \in R_{0,+}$, since this would imply that $u^{-1}(\beta)=-\alpha \in R_{-}$. We have shown that $u(\alpha) \in R_{+}$in both cases, that is $u \in U$ as required.

We prove 2. By definition of $U$ we have $\bar{R}_{+} \subset \bigcap_{u \in U} u^{-1}\left(R_{+}\right)$. Assume $\alpha \in \bigcap_{u \in U} u^{-1}\left(R_{+}\right)$. We then have $u(\alpha) \in R_{+}$for any $u \in U$. Consider $\gamma \in \bar{P}_{+}$. By assertion 1 , there exists $u \in U$ such that $\gamma \in u^{-1}\left(P_{+}\right)$. We thus have $\left(\gamma, \alpha^{\vee}\right)=\left(u(\gamma), u(\alpha)^{\vee}\right) \geq 0$ for any $\gamma \in \bar{P}_{+}$. This implies that $\alpha$ is a positive root of $\bar{R}_{+}$.

We prove 3. Recall that the stabilizer of $\rho$ under $W$ reduces to $\{1\}$. Consider $w \in W$. There exists $\bar{w} \in \bar{W}$ such that $\bar{w}\left(w^{-1} \cdot \rho\right) \in \bar{P}_{+}$. By assertion 1 , there exists $u \in U$ such that $u \bar{w}\left(w^{-1} \cdot \rho\right) \in P_{+}$. Since $\rho$ is the unique element of the orbit $W \cdot \rho$ in $P_{+}$, we must have $w=u \bar{w}$. Now assume 
that there exist $u_{1}, u_{2} \in U$ and $\bar{w}_{1}, \bar{w}_{2} \in \bar{W}$ such that $u_{1} \bar{w}_{1}=u_{2} \bar{w}_{2}$. We have $u_{2}=u_{1} \bar{w}$ with $\bar{w}=\bar{w}_{1} \bar{w}_{2}^{-1} \in \bar{W}$. If $\bar{w} \neq 1$, there exists $\alpha \in \bar{R}_{+}$such that $\bar{w}(\alpha)=-\beta$ with $\beta \in \bar{R}_{+}$. Then $\left(\rho, u_{2}(\alpha)^{\vee}\right)=-\left(\rho, u_{1}(\beta)^{\vee}\right)<0$ since $u_{1}(\beta) \in R_{+}$. This contradicts the hypothesis $u_{2}(\alpha) \in R_{+}$. hence $\bar{w}=1$, that is $\bar{w}_{1}=\bar{w}_{2}$ and $u_{1}=u_{2}$.

Denote by $\bar{E}$ the $\mathbb{Q}$-vector space generated by the roots in $\bar{R}_{+}$. Then we have $\bar{E} \cap R_{+}=\bar{R}_{+}$; see [4. §1.10]. We will make frequent use of this fact in the rest of the paper. It is important to notice that this holds because we assumed that $\bar{S} \subset S$.

Lemma 3.2. Let $u \in U$. Then $u(\bar{\rho})=\bar{\rho}$ if and only if $u\left(\bar{R}_{+}\right)=\bar{R}_{+}$.

Proof. Assume that there exists $\alpha \in \bar{R}_{+}$such that $u(\alpha) \notin \bar{R}_{+}$. Then since $u(\alpha) \in R_{+}$we have $u(\alpha) \notin \bar{E}$. It follows that there exists a simple root $\alpha_{j} \notin \bar{R}_{+}$such that $u(\alpha) \geq \alpha_{j}$. As $u\left(\bar{R}_{+}\right) \subset R_{+}$, there can't be any cancellation of simple roots when decomposing $u(\bar{\rho})$ on the basis $S$. Therefore we have $u(\bar{\rho}) \geq \alpha_{j}$ and $u(\bar{\rho}) \notin \bar{E}$. From there, we see that we cannot have $u(\bar{\rho})=\bar{\rho}$ since $\bar{\rho} \in \bar{E}$. The converse is trivial.

Lemma 3.3. Let $u \in U$ be such that $u(\bar{\rho}) \neq \bar{\rho}$. Then $u(\bar{\rho}) \nless \bar{\rho}$.

Proof. Since $u\left(\bar{R}_{+}\right) \neq \bar{R}_{+}$, arguing as in the proof of the previous lemma, we know that there exists $\alpha \in \bar{R}_{+}$and a simple root $\alpha_{j} \notin \bar{E}$ such that $u(\alpha) \geq \alpha_{j}$. Since $\bar{\rho} \in \bar{E}$, the root $\alpha_{j}$ appears in the decomposition of $u(\bar{\rho})-\bar{\rho}$ in the basis $S$ with a positive coefficient hence we cannot have $u(\bar{\rho})<\bar{\rho}$.

Lemma 3.4. Let $\gamma, \gamma^{\prime} \in P$ be such that $\gamma \leq_{\bar{R}_{+}} \gamma^{\prime}$. Then we have $u(\gamma) \leq_{R_{+}} u\left(\gamma^{\prime}\right)$ for all $u \in U$.

Proof. By definition $\gamma \geq \bar{R}_{+} \gamma^{\prime}$ implies that $\gamma-\gamma^{\prime}$ is a sum of roots in $\bar{R}_{+}$. Since $u\left(\bar{R}_{+}\right) \subset R_{+}$we see that $u\left(\gamma-\gamma^{\prime}\right)$ is a sum of roots in $R_{+}$. Hence $u\left(\gamma-\gamma^{\prime}\right)=u(\gamma)-u\left(\gamma^{\prime}\right) \geq_{R_{+}} 0$ as required.

Lemma 3.5. Let $\gamma \in P$ be such that $\gamma \notin \bar{P}_{+}$. Then we have $u(\gamma) \notin P_{+}$for all $u \in U$.

Proof. Since $\gamma \notin \bar{P}_{+}$, there exists $\alpha \in \bar{R}_{+}$such that $\left(\gamma, \alpha^{\vee}\right)<0$. It follows that

$$
\left(u(\gamma), u(\alpha)^{\vee}\right)=\left(\gamma, \alpha^{\vee}\right)<0 .
$$

Since $u(\alpha) \in R_{+}$, this implies that $u(\gamma) \notin P_{+}$.

\section{INDUCED CHARACTERS}

4.1. The functions $H_{\mu}$. Given $\mu \in \bar{P}_{+}$, write $H_{\mu}:=\operatorname{char}(V(\mu) \uparrow \mathfrak{g})$ the induced character of $\bar{V}(\mu)$ from $\overline{\mathfrak{g}}$ to $\mathfrak{g}$. We then have

$$
H_{\mu}:=\sum_{\lambda \in P_{+}} m_{\mu}^{\lambda} s_{\lambda}
$$

Observe there can exist infinitely many weights $\lambda$ such that $m_{\mu}^{\lambda} \neq 0$. When $\overline{\mathfrak{g}}=\mathfrak{h}$ is reduced to the Cartan subalgebra, we have $\bar{R}_{+}=\emptyset$ and we set $m_{\lambda}^{\mu}=K_{\lambda, \mu}=\operatorname{dim} V(\lambda)_{\mu}$ so that

$$
h_{\mu}:=\sum_{\lambda \in P_{+}} K_{\lambda, \mu} s_{\lambda} .
$$

Since $K_{\lambda, \mu}=K_{\lambda, w(\mu)}$ for any $w \in W$, we have $h_{\mu}=h_{w(\mu)}$ (for the usual action of $W$ on $P$ ). Moreover, $K_{\mu, \mu}=1$ and $K_{\lambda, \mu} \neq 0$ if and only if $\lambda \geq \mu$ (i.e. $\lambda-\mu$ decomposes as a sum of simple roots). The sets $\left\{s_{\lambda} \mid \lambda \in P_{+}\right\}$and $\left\{h_{\lambda} \mid \lambda \in P_{+}\right\}$are bases of $\mathbb{G}$ and the corresponding transition matrix is unitriangular for the order $\leq$. 
We now define two $\mathbb{Z}$-linear maps $H$ and $S$ by

$$
H:\left\{\begin{array}{c}
\mathbb{Z}[P] \rightarrow \mathbb{G} \\
e^{\beta} \mapsto h_{\beta}
\end{array} \text { and } S:\left\{\begin{array}{c}
\mathbb{Z}[P] \rightarrow \mathbb{G} \\
e^{\beta} \mapsto s_{\beta}
\end{array}\right.\right.
$$

and we set

$$
\Delta=\prod_{\alpha \in R_{+}}\left(1-e^{\alpha}\right)
$$

Proposition 4.1. The maps $H$ and $S$ satisfy the relations

$$
S\left(e^{\beta}\right)=H\left(\Delta e^{\beta}\right) \text { and } H\left(e^{\beta}\right)=S\left(\Delta^{-1} e^{\beta}\right)
$$

for any $\beta \in P$. Therefore $S=H \circ \Delta$ and $H=S \circ \Delta^{-1}$ (by writing for short $\Delta$ and $\Delta^{-1}$ for the multiplication by $\Delta$ and $\Delta^{-1}$ in $\left.\mathbb{Z}[[P]]\right)$.

Proof. The partition function $\mathcal{P}$ is defined by

$$
\Delta^{-1}=\prod_{\alpha \in R_{+}} \frac{1}{1-e^{\alpha}}=\sum_{\gamma \in P} \mathcal{P}(\gamma) e^{\gamma}
$$

and we have by definition $h_{\beta}=\sum_{\lambda} K_{\lambda, \beta} s_{\lambda}$ where $K_{\lambda, \beta}=\sum_{w} \varepsilon(w) \mathcal{P}(w \circ \lambda-\beta)$. This gives

$$
S\left(\Delta^{-1} e^{\beta}\right)=\sum_{\gamma \in P} \mathcal{P}(\gamma) s_{\beta+\gamma} .
$$

Let $\gamma \in P$. Then either $s_{\beta+\gamma}=0$ or there exists $\lambda \in P_{+}$and $w \in W$ such that $w^{-1} \circ(\beta+\gamma)=\lambda$, that is $\gamma=w \circ \lambda-\beta$. This yields $s_{\beta+\gamma}=\varepsilon(w) s_{\lambda}$ and in turn we obtain

$$
S\left(\Delta^{-1} e^{\beta}\right)=\sum_{\lambda} \sum_{w \in W} \varepsilon(w) \mathcal{P}(w \circ \lambda-\beta) s_{\lambda}=\sum_{\lambda} K_{\lambda, \beta} s_{\lambda}=h_{\beta}
$$

as desired. Note that we have for any $U \in \mathbb{Z}[P], H(U):=S\left(\Delta^{-1} U\right)$. Then if we set $U=\Delta e^{\beta}$, we get the relation $H\left(\Delta e^{\beta}\right)=S\left(e^{\beta}\right)$, as required.

Now write $\overline{\mathbb{G}}=\mathbb{Z}^{\bar{W}}\left[e^{\beta} \mid \beta \in P\right]$ the character ring of $\overline{\mathfrak{g}}$ (polynomials of $\mathbb{Z}[P]$ invariant under the action of $\bar{W}$ the Weyl group of $\overline{\mathfrak{g}}$ ). The set of irreducible characters $\left\{\bar{s}_{\mu} \mid \mu \in \bar{P}_{+}\right\}$of $\overline{\mathfrak{g}}$ is a basis of $\overline{\mathbb{G}}$. Define the $\mathbb{Z}$-linear map

and set

$$
\bar{H}:\left\{\begin{array}{c}
\mathbb{Z}[P] \rightarrow \mathbb{G} \\
e^{\mu} \mapsto H_{\mu}
\end{array}\right.
$$

$$
\bar{\Delta}=\prod_{\alpha \in R_{+} \backslash \bar{R}_{+}}\left(1-e^{\alpha}\right) \text { and } \bar{\nabla}=\prod_{\alpha \in \bar{R}_{+}}\left(1-e^{\alpha}\right) \text {. }
$$

\section{Proposition 4.2.}

(1) The maps $\bar{H}$ and $S$ satisfy the relation

$$
\bar{H}\left(e^{\mu}\right)=S\left(\bar{\Delta}^{-1} e^{\mu}\right)
$$

for any $\mu \in P$. We write for short $\bar{H}=S \circ \bar{\Delta}^{-1}$.

(2) We have $\bar{H}\left(e^{\mu}\right)=H\left(\bar{\nabla} e^{\mu}\right)$.

Proof. The first assertion is proved as in the previous proof by replacing the partition function $\mathcal{P}$ by $\overline{\mathcal{P}}$. For the second one, we combine the first part with the previous proposition. 
We have, using the Weyl character formula:

$$
\bar{\nabla}=\prod_{\alpha \in \bar{R}_{+}}\left(1-e^{\alpha}\right)=\sum_{\bar{w} \in \bar{W}} \varepsilon(\bar{w}) e^{\bar{\rho}-\bar{w}(\bar{\rho})}
$$

where $\bar{\rho}$ is the half sum of positive roots of $\overline{\mathfrak{g}}$. By the second assertion of the previous proposition, we get for all $\mu \in P$

$$
H_{\mu}=\bar{H}\left(e^{\mu}\right)=\sum_{\bar{w} \in \bar{W}} \varepsilon(\bar{w}) h_{\mu+\bar{\rho}-\bar{w}(\bar{\rho})} .
$$

4.2. Irreducible components of $\bar{R}$. Now assume the semisimple Lie algebra $\overline{\mathfrak{g}}$ has a decomposition of the form

$$
\overline{\mathfrak{g}}=\mathfrak{g}_{1} \oplus \mathfrak{g}_{2} \oplus \cdots \oplus \mathfrak{g}_{r}
$$

where each $\mathfrak{g}_{k}, k=1, \ldots, r$ is a Lie subalgebra of $\mathfrak{g}$ with irreducible root system $R_{k} \subset \bar{R}$ and $\bar{R}=\bigsqcup_{k=1}^{r} R^{(k)}$. We also assume that we have $P=P^{(1)} \oplus \cdots \oplus P^{(r)}$ where $P^{(k)}$ is the weight lattice of $\mathfrak{g}_{k}$. In particular each weight $\mu \in P_{+}$decomposes on the form $\mu=\mu^{(1)}+\cdots+\mu^{(r)}$ with $\mu^{(k)} \in P_{+}^{(k)}$. We then have additional properties for the functions $H_{\mu}$ we shall need in Section 7.1

We have

$$
\bar{\nabla}=\prod_{\alpha \in \bar{R}_{+}}\left(1-e^{\alpha}\right)=\prod_{k=1}^{r} \prod_{\alpha \in R_{+}^{(k)}}\left(1-e^{\alpha}\right)
$$

and

$$
H_{\mu}=\prod_{k=1}^{r} \prod_{\alpha \in R_{+}^{(k)}}\left(1-e^{\alpha}\right) h_{\mu^{(1)}+\cdots+\mu^{(r)}} .
$$

Combining (11) and Proposition 4.1 (for each root system $R_{k}$ ), we get for any $k=1, \ldots, r$,

$$
\prod_{\alpha \in R_{+}^{(k)}}\left(1-e^{\alpha}\right) h_{\mu^{(1)}+\cdots+\mu^{(r)}}=\sum_{\lambda^{(k)} \in P_{+}^{(k)}} K_{\lambda^{(k)}, \mu^{(k)}}^{-1} h_{\mu^{(1)}+\cdots \lambda^{(k)}+\cdots+\mu^{(r)}}
$$

where the coefficients $K_{\lambda^{(k)}, \mu^{(k)}}^{-1}$ are those of the inverse matrix of $\left(K_{\lambda^{(k)}, \mu^{(k)}}\right)_{\lambda^{(k)}, \mu^{(k)} \in P_{+}^{(k)}}$. By an easy induction, we then get

$$
H_{\mu}=\sum_{\lambda^{(1)} \in P_{+}^{(k)}} \cdots \sum_{\lambda^{(r)} \in P_{+}^{(r)}} K_{\lambda^{(1)}, \mu^{(1)}}^{-1} \cdots K_{\lambda^{(r)}, \mu^{(r)}}^{-1} h_{\lambda^{(1)}+\cdots+\lambda^{(r)}} .
$$

4.3. The conjecture. We start with an easy observation.

Lemma 4.3. Consider $u \in W$. Then the two following statements are equivalent:

(1) $u\left(\bar{R}_{+}\right)=\bar{R}_{+}$

(2) $u$ is a Dynkin diagram automorphism of $\overline{\mathfrak{g}}$

Proof. When $u$ is a Dynkin diagram automorphism of $\overline{\mathfrak{g}}$, we clearly have $u\left(\bar{R}_{+}\right)=\bar{R}_{+}$. Now assume $u\left(\bar{R}_{+}\right)=\bar{R}_{+}$. Then we have $u(\bar{R})=\bar{R}$ and $u$ is an automorphism of the root system $\bar{R}$. It is known that $\operatorname{Aut}(\bar{R})=\bar{W} \ltimes \operatorname{Aut}(\bar{\Gamma})$ where $\bar{\Gamma}$ is the Dynkin diagram of $\bar{R}$ i.e. $\operatorname{Aut}(\bar{R})$ is the semidirect product of $\bar{W}$ (which is normal in $\operatorname{Aut}(\bar{R})$ ) with $\operatorname{Aut}(\bar{\Gamma})$. Since $u\left(\bar{R}_{+}\right)=\bar{R}_{+}$the element $u$ belongs in fact in $\operatorname{Aut}(\bar{\Gamma})$ (otherwise $u$ would send at least a positive root of $\bar{R}_{+}$on a negative root). 
Proposition 4.4. Let $\mu, \nu \in \bar{P}_{+}$. Assume that there exists $u \in W$ such that $u\left(\bar{R}_{+}\right)=\bar{R}_{+}$and $\nu=u(\mu)$ (or equivalently, $\mu$ and $\nu$ are conjugate by a Dynkin diagram automorphism of $\overline{\mathfrak{g}}$ lying in the Weyl group of $\mathfrak{g})$. Then $H_{\mu}=H_{\nu}$.

Proof. With the previous notation, we have

$$
H_{\mu}=H\left(\prod_{\alpha \in \bar{R}_{+}}\left(1-e^{\alpha}\right) e^{\mu}\right) \text { and } H_{\nu}=H\left(\prod_{\alpha \in \bar{R}_{+}}\left(1-e^{\alpha}\right) e^{\nu}\right)
$$

Since $u\left(\bar{R}_{+}\right)=\bar{R}_{+}$we see that $u(\bar{\rho})=\bar{\rho}$ and that $u \bar{W} u^{-1}=\bar{W}$ (indeed, $u s_{\alpha} u^{-1}=s_{u \alpha}$ for all $\alpha \in \bar{R})$. Therefore

$$
\prod_{\alpha \in \bar{R}_{+}}\left(1-e^{\alpha}\right) e^{\nu}=\sum_{w \in \bar{W}} \varepsilon(w) e^{\nu+\bar{\rho}-w(\bar{\rho})}=\sum_{w \in \bar{W}} \varepsilon(w) e^{u(\mu)+u(\bar{\rho})-u w\left(u^{-1}(\bar{\rho})\right)}=\sum_{w \in \bar{W}} \varepsilon(w) e^{u(\mu+\bar{\rho}-w(\bar{\rho}))} .
$$

It follows that

$$
H_{\nu}=H\left(\sum_{w \in \bar{W}} \varepsilon(w) e^{u(\mu+\bar{\rho}-w(\bar{\rho}))}\right)=\sum_{w \in \bar{W}} \varepsilon(w) h_{u(\mu+\bar{\rho}-w(\bar{\rho}))}=\sum_{w \in \bar{W}} \varepsilon(w) h_{\mu+\bar{\rho}-w(\bar{\rho})}=H_{\mu}
$$

since $h_{w(\beta)}=h_{\beta}$ for any $w \in W$.

We conjecture that the converse is true:

Conjecture 4.5. Consider $\mu, \nu \in \bar{P}_{+}$. Then we have $H_{\mu}=H_{\nu}$ if and only if there exists $u$ in $W$ such that $u\left(\bar{R}_{+}\right)=\bar{R}_{+}$and $\nu=u(\mu)$ or equivalently, $\mu$ and $\nu$ are conjugate by a Dynkin diagram automorphism of $\overline{\mathfrak{g}}$ lying in the Weyl group of $\mathfrak{g}$.

\section{Triangular decomposition of $H_{\mu}$}

5.1. Decomposition on the $h$-basis. Let $\mu \in \bar{P}_{+}$and let $w \in U$ be such that $\mu \in \bar{C}_{w}=w^{-1} \bar{C}$. Recall that $R_{+}^{w}=w^{-1}\left(R_{+}\right)$. Since $w \in U$, we have that $w\left(\bar{R}_{+}\right) \subset R_{+}$which in turn implies that $\bar{R}_{+} \subset R_{+}^{w}$, that is $\preceq \subset \leq_{w}$.

Proposition 5.1. Let $w \in U$. We have for all $\mu \in \bar{P}_{+}$

$$
H_{\mu}=h_{\mu}+\sum_{\lambda \in P_{+}^{w}, \mu<{ }_{w} \lambda} a_{\lambda, \mu} h_{\lambda}
$$

where for any $\lambda \in P_{+}^{w}$

$$
a_{\lambda, \mu}=\sum_{\bar{w} \in \bar{W} \mid \mu+\bar{\rho}-\bar{w}(\bar{\rho}) \in W \cdot \lambda} \varepsilon(\bar{w})
$$

Proof. Since $\preceq \subset \leq_{w}$, we have

$$
H_{\mu}=h_{\mu}+\sum_{\bar{w} \in \bar{W}, \bar{w} \neq 1} \varepsilon(\bar{w}) h_{\mu+\bar{\rho}-\bar{w}(\bar{\rho})} \text { with } \mu<_{w} \mu+\bar{\rho}-\bar{w}(\bar{\rho}) \text { for } \bar{w} \neq 1
$$

Now for each $w \neq 1$, the orbit of each $\gamma=\mu+\bar{\rho}-\bar{w}(\bar{\rho})$ intersects $P_{+}^{w}$ at one point (say $\lambda$ ) and we can use the relations $h_{w(\gamma)}=h_{\gamma}$ for any $w \in W$. Moreover, we then have $\gamma \leqslant w \lambda$. We thus obtain $\mu<_{w} \mu+\bar{\rho}-\bar{w}(\bar{\rho}) \leqslant_{w} \lambda$ which gives the unitriangularity of the decomposition. The coefficients $a_{\lambda, \mu}$ are then obtained by gathering the contributions in $h_{\lambda}$ for each $\lambda \in P_{+}^{w}$. 
Remark 5.2. (1) For $\mathfrak{g}=\overline{\mathfrak{g}}$, the coefficients $a_{\lambda, \mu}$ are the entries of the inverse matrix $K^{-1}$ where $K=\left(K_{\lambda, \mu}\right)_{\lambda, \mu \in P_{+}}$. In type $A, K$ is the Kostka matrix. Obtaining a combinatorial formula for the coefficients of $K^{-1}$ is already a nontrivial problem (see [2] and the references therein). As far as we are aware no such description for the coefficients of $K^{-1}$ exists for other root systems (and thus also for the coefficients $a_{\lambda, \mu}$ associated to a general Levi subalgebra).

(2) We can also deduce from Propositions 3.1 and 5.1 that for any $u \in U$, the set $\left\{H_{\lambda} \mid \lambda \in P_{+}^{u}\right\}$ is a basis of $\mathbb{G}$.

\subsection{Consequences.}

Proposition 5.3. Let $\mu$ and $\nu$ be dominant weights in $\bar{P}_{+}$such that $H_{\mu}=H_{\nu}$. Then, there exists $\tau \in W$ such that $\tau(\nu)=\mu$. In particular, if $\mu$ and $\nu$ belong to the same closed Weyl chamber for $\mathfrak{g}$, we have $\tau=1$ and $\mu=\nu$.

Proof. Assume that $\mu$ belongs to $\bar{P}_{+}^{w}$ and $\nu$ belongs to $\bar{P}_{+}^{w^{\prime}}$ with $w, w^{\prime}$ in $U$. Let $\tau \in W$ be such that $w^{\prime}=w \tau$. We then have $R_{+}^{w^{\prime}}=\tau^{-1}\left(R_{+}^{w}\right)$ and $P_{+}^{w^{\prime}}=\tau^{-1}\left(P_{+}^{w}\right)$. Moreover $\mu<_{w} \gamma$ if and only if $\tau^{-1}(\mu)<_{w^{\prime}} \tau^{-1}(\gamma)$. On the one hand, using Proposition 5.1, we get

$$
\begin{aligned}
H_{\nu} & =h_{\nu}+\sum_{\lambda \in P_{+}^{w w^{\prime}, \nu<w^{\prime} \lambda}} a_{\lambda, \nu} h_{\lambda} \\
& =h_{\nu}+\sum_{\lambda \in P_{+}^{w}, \tau(\nu)<_{w} \lambda} a_{\tau^{-1}(\lambda), \nu} h_{\tau^{-1}(\lambda)} .
\end{aligned}
$$

Since $h_{w(\beta)}=h_{\beta}$ for all $w \in W$ and $\beta \in P$, this can be rewritten under the form

$$
H_{\nu}=h_{\tau(\nu)}+\sum_{\lambda \in P_{+}^{w}, \tau(\nu)<w \lambda} a_{\tau^{-1}(\lambda), \nu} h_{\lambda} .
$$

On the other hand we have

$$
H_{\mu}=h_{\mu}+\sum_{\lambda \in P_{+}^{w}, \mu<w \lambda} a_{\lambda, \mu} h_{\lambda} .
$$

So $H_{\nu}=H_{\mu}$ implies that $h_{\tau(\nu)}=h_{\mu}$ by comparing the indices of the basis vectors of $\left\{h_{\lambda} \mid \lambda \in P_{+}^{w}\right\}$ which are minimal for the order $\leq_{w}$. Hence $\mu=\tau(\nu)$ as desired.

Remark 5.4. If $H_{\mu}=H_{0}$ (i.e. we have $\nu=0$ ), then $\mu=0$ since $\mu$ et 0 always belong to the same closed Weyl chamber.

For any weight $\mu \in \bar{P}_{+}$, define the set $E_{\mu}=\{\mu+\bar{\rho}-\bar{w}(\bar{\rho}) \mid \bar{w} \in \bar{W}\}$. Since the stabilizer of $\bar{\rho}$ under the action of $\bar{W}$ reduces to $\{1\}$, the cardinality of $E_{\mu}$ is equal to that of $\bar{W}$. The following corollary shows that the conjecture holds when each of the sets $E_{\mu}$ and $E_{\nu}$ is contained in a closed Weyl chamber. This happens in particular when $\mu$ and $\nu$ are sufficiently far from the walls of the Weyl chambers in which they appear.

Corollary 5.5. Let $\mu$ and $\nu$ be two dominant weights in $\bar{P}_{+}$. Assume that there exist $w \in W$ such that $E_{\mu} \subset P_{+}^{w}$ and $w^{\prime} \in W$ such that $E_{\nu} \subset P_{+}^{w^{\prime}}$. Then $H_{\mu}=H_{\nu}$ implies that $\nu=\tau(\mu)$ and $\tau\left(\bar{R}_{+}\right)=\bar{R}_{+}$with $\tau \in W$ such that $w^{\prime}=w \tau$.

Proof. All the elements of $E_{\mu}$ belong to $P_{+}^{w}$. They thus belong to distinct $W$-orbits. Hence the decomposition of $H_{\mu}$ in the basis $\left\{h_{\lambda} \mid \lambda \in P_{+}^{w}\right\}$ is

$$
H_{\mu}=h_{\mu}+\sum_{\bar{w} \in \bar{W}, \bar{w} \neq 1} \varepsilon(\bar{w}) h_{\mu+\bar{\rho}-\bar{w}(\bar{\rho})} .
$$


Similarly, the elements of $E_{\nu}$ belong to distinct $W$-orbits. Hence the decomposition of $H_{\nu}$ in the basis $\left\{h_{\lambda} \mid \lambda \in P_{+}^{w^{\prime}}\right\}$ is

$$
H_{\nu}=h_{\nu}+\sum_{\bar{w}^{\prime} \in \bar{W}, \bar{w}^{\prime} \neq 1} \varepsilon\left(\bar{w}^{\prime}\right) h_{\nu+\bar{\rho}-\bar{w}^{\prime}(\bar{\rho})} .
$$

Since $H_{\nu}=H_{\mu}$, we see that there exists $\tau \in W$ such that $\tau(\nu)=\mu$ by the previous proposition. Further, we know that $\tau$ is such that $P_{+}^{w^{\prime}}=\tau^{-1}\left(P_{+}^{w}\right)$ thus we have $\tau\left(E_{\nu}\right)=E_{\mu}$. Let $\alpha \in \bar{R}_{+}$ and $\bar{w}=s_{\alpha}$. Then $\bar{w}(\bar{\rho})-\bar{\rho}=\alpha$ and we see that there exists an element $\bar{w}^{\prime} \in \bar{W}$ such that $\tau(\nu+\alpha)=\mu+\bar{\rho}-\bar{w}^{\prime}(\bar{\rho})$. In turn, this implies $\tau(\alpha)=\bar{\rho}-\bar{w}^{\prime}(\bar{\rho})$ as $\tau(\nu)=\mu$ and $\tau(\alpha)$ is a sum of positive roots in $\bar{R}_{+}$. But $\tau(\alpha)$ also lies in $R$, hence $\tau(\alpha) \in \bar{R}_{+}$; see Section 3. We have shown that $\tau$ maps $\bar{R}_{+}$onto itself as expected.

\section{The FunCtions $M_{\mu}$}

We know give an equivalent formulation of our problem in terms of parabolic analogues of monomial functions.

6.1. Decomposition on the monomial functions. For any weight $\gamma \in P$, set $\mathrm{m}_{\gamma}=\sum_{w \in W} e^{w(\gamma)}$ so that $\left.\mathrm{m}_{\gamma}\right]^{1}$ is the image of $e^{\gamma}$ by the symmetrization operator

$$
\mathcal{M}:\left\{\begin{array}{c}
\mathbb{Z}[P] \rightarrow \mathbb{Z}[P]^{W} \\
e^{\gamma} \mapsto \mathrm{m}_{\gamma}
\end{array}\right.
$$

We clearly have $\mathrm{m}_{w(\gamma)}=\mathrm{m}_{\gamma}$ for any $w \in W$. Also $\left\{\mathrm{m}_{\lambda} \mid \lambda \in P_{+}^{w}\right\}$ is a basis of $\mathbb{G}$. Given $\mu \in P$, set

$$
M_{\mu}:=\mathcal{M}\left(\prod_{\alpha \in \bar{R}_{+}}\left(1-e^{\alpha}\right) e^{\mu}\right)=\sum_{\bar{w} \in \bar{W}} \varepsilon(\bar{w}) \mathrm{m}_{\mu+\bar{\rho}-\bar{w}(\bar{\rho})} .
$$

\section{Lemma 6.1.}

(1) We have

$$
M_{\mu}=\sum_{\lambda \in P_{+}} a_{\lambda, \mu} \mathrm{m}_{\lambda} \text { with } a_{\lambda, \mu}=\sum_{\bar{w} \in \bar{W} \mid \mu+\bar{\rho}-\bar{w}(\bar{\rho}) \in W \cdot \lambda} \varepsilon(\bar{w}) .
$$

(2) Consider $\mu, \nu \in \bar{P}_{+}$. Then $H_{\mu}=H_{\nu}$ if and only if $M_{\mu}=M_{\nu}$.

Proof. Assertion 1 follows from the identity $\mathrm{m}_{w(\gamma)}=\mathrm{m}_{\gamma}$ for any $\gamma \in P$ and any $w \in W$. By Proposition 5.1, the coefficients of the expansion of $M_{\mu}$ on the basis $\left\{\mathrm{m}_{\lambda} \mid \lambda \in P_{+}\right\}$are the same as those appearing in the expansion of $H_{\mu}$ on the basis $\left\{h_{\lambda} \mid \lambda \in P_{+}^{w}\right\}$. Assertion 2 follows.

6.2. A simple expression for the functions $M_{\lambda}$. For any $\gamma \in P$, set

$$
\bar{a}_{\gamma}=\sum_{\bar{w} \in \bar{W}} \varepsilon(\bar{w}) e^{\bar{w}(\gamma)}
$$

We thus have $\bar{a}_{\bar{w}(\gamma)}=\varepsilon(\bar{w}) \bar{a}_{\gamma}$ and $\bar{w}\left(\bar{a}_{\gamma}\right)=\varepsilon(\bar{w}) \bar{a}_{\gamma}$ for any $\bar{w} \in \bar{W}$ and $\bar{a}_{\bar{w}_{0}(\bar{\rho})}=\varepsilon\left(\bar{w}_{0}\right) \bar{a}_{\bar{\rho}}$ where $\bar{w}_{0}$ is the element of maximal length in $\bar{W}$.

Proposition 6.2. Let $\mu \in \bar{P}_{+}$.

(1) We have

$$
M_{\mu}=\varepsilon\left(\bar{w}_{0}\right) \sum_{u \in U} u\left(\bar{a}_{\mu+\bar{\rho}} \cdot \bar{a}_{\bar{\rho}}\right)
$$

\footnotetext{
${ }^{1}$ Our function $\mathrm{m}_{\gamma}$ slightly differs from the usual monomial function $m_{\gamma}=\frac{1}{\left|W_{\gamma}\right|} \sum_{w \in W} e^{w(\gamma)}$ where $W_{\gamma}$ is the stabilizer of $\gamma$ under the action of $W$.
} 
(2) Let $\Lambda$ be the unique element lying in $\{u(\mu+2 \bar{\rho}) \mid u \in U\} \cap P_{+}$. Then we have

$$
M_{\mu}=\varepsilon\left(\bar{w}_{0}\right) e^{\Lambda}+\sum_{\gamma \in P, \gamma<\Lambda} b_{\lambda, \mu} e^{\gamma} .
$$

Proof. We prove (1). We have

$$
M_{\mu}=\sum_{\bar{w} \in \bar{W}} \varepsilon(\bar{w}) \mathrm{m}_{\mu+\bar{\rho}-\bar{w}(\bar{\rho})}=\sum_{w \in W} w\left(e^{\mu+\bar{\rho}} \sum_{\bar{w} \in \bar{W}} \varepsilon(\bar{w}) e^{-\bar{w}(\bar{\rho})}\right) .
$$

This gives

$$
M_{\mu}=\sum_{w \in W} w\left(e^{\mu+\bar{\rho}} \bar{a}_{-\bar{\rho}}\right)=\varepsilon\left(\bar{w}_{0}\right) \sum_{w \in W} w\left(e^{\mu+\bar{\rho}} \bar{a}_{\bar{\rho}}\right)=\varepsilon\left(\bar{w}_{0}\right) \sum_{u \in U} u\left(\sum_{\bar{w} \in \bar{W}} \bar{w}\left(e^{\mu+\bar{\rho}} \bar{a}_{\bar{\rho}}\right)\right)
$$

by using Assertion 3 of Proposition 3.1. Hence

$$
\begin{aligned}
M_{\mu} & =\varepsilon\left(\bar{w}_{0}\right) \sum_{u \in U} u\left(\sum_{\bar{w} \in \bar{W}} e^{\bar{w}(\mu+\bar{\rho})} \bar{w}\left(\bar{a}_{\bar{\rho}}\right)\right) \\
& =\varepsilon\left(\bar{w}_{0}\right) \sum_{u \in U} u\left(\bar{a}_{\bar{\rho}} \sum_{\bar{w} \in \bar{W}} \varepsilon(\bar{w}) e^{\bar{w}(\mu+\bar{\rho})}\right) \\
& =\varepsilon\left(\bar{w}_{0}\right) \sum_{u \in U} u\left(\bar{a}_{\mu+\bar{\rho}} \cdot \bar{a}_{\bar{\rho}}\right)
\end{aligned}
$$

since $\bar{a}_{\bar{w}(\bar{\rho})}=\varepsilon(\bar{w}) \bar{a}_{\bar{\rho}}$.

We prove (2). The monomials $e^{\mu+\bar{\rho}}$ and $e^{\bar{\rho}}$ are the monomials of highest weight (with respect to $\leq_{\bar{R}_{+}}$) appearing in the expression of $\bar{a}_{\mu+\bar{\rho}}$ and $\bar{a}_{\bar{\rho}}$ respectively. It follows that the monomial $e^{\mu+2 \bar{\rho}}$ is of highest weight among those appearing in $\bar{a}_{\mu+\bar{\rho}} \cdot \bar{a}_{\bar{\rho}}$. Thus using (1) we get an expression of the form

$$
M_{\mu}=\varepsilon\left(\bar{w}_{0}\right) \sum_{u \in U} u\left(e^{\mu+2 \bar{\rho}}+\sum_{\nu<\bar{R}_{+} \mu+2 \bar{\rho}} \mathbb{Z} e^{\nu}\right) .
$$

By Lemma 3.4 $\nu<\bar{R}_{+} \mu+2 \bar{\rho}$ implies that $u(\nu)<u(\mu+2 \bar{\rho})$. Finally, the maximal weight with respect to $\leq$ in the set $\{u(\mu+2 \bar{\rho}) \mid u \in U\}$ is the unique element $\Lambda$ lying in $\{u(\mu+2 \bar{\rho}) \mid u \in U\} \cap P_{+}$. Therefore we have

$$
M_{\mu}=\varepsilon\left(\bar{w}_{0}\right) e^{\Lambda}+\sum_{\gamma \in P, \gamma<\Lambda} b_{\lambda, \mu} e^{\gamma}
$$

as required.

\subsection{Proof of the conjecture for $\mu+2 \bar{\rho}$ dominant.}

Lemma 6.3. Let $\mu \in \bar{P}_{+}$be such that $\mu+2 \bar{\rho}$ belongs to $P_{+}$. Then $\mu \in P_{+}$.

Proof. For any simple root $\alpha_{i} \in S$, we have $\left(\mu+2 \bar{\rho}, \alpha_{i}^{\vee}\right) \geq 0$ since $\mu+2 \bar{\rho} \in P_{+}$. Also for any simple root $\alpha_{i} \in \bar{S}$, we have $\left(\mu, \alpha_{i}^{\vee}\right) \geq 0$ since $\mu \in \bar{P}_{+}$. Now consider $\alpha_{j} \in S \backslash \bar{S}$. Since $2 \bar{\rho}$ decomposes as a sum of simple roots in $\bar{S}$, we must have $\left(2 \bar{\rho}, \alpha_{j}^{\vee}\right) \leq 0$. Indeed for any $\alpha_{i} \in \bar{S},\left(\alpha_{i}, \alpha_{j}^{\vee}\right)=0$ or is negative as it can be easily seen by considering the the entries of the Cartan matrix of $\mathfrak{g}$ which do not appear on the diagonal. Therefore $\left(\mu, \alpha_{j}^{\vee}\right) \geq\left(\mu+2 \bar{\rho}, \alpha_{j}^{\vee}\right) \geq 0$. 
Proposition 6.4. Let $\mu, \nu \in \bar{P}_{+}$be such that $H_{\mu}=H_{\nu}$ and assume that $\mu+2 \bar{\rho} \in P_{+}$. Then, there exists $v \in U$ such that $\nu=v(\mu)$ and $v\left(\bar{R}_{+}\right)=\bar{R}_{+}$.

Proof. By the previous lemma, we see that $\mu \in P_{+}$. Let $v \in U$ be such that $\nu \in P_{+}^{v}$. Then by (the proof of) Proposition 5.3. we know that $v(\nu)=\mu$. Next Lemma 6.1 implies that $M_{\mu}=M_{\nu}$ and, in particular, $M_{\mu}$ and $M_{\nu}$ have the same maximal monomial with respect to $<$. Hence

$$
\{u(\mu+2 \bar{\rho}) \mid u \in U\} \cap P_{+}=\left\{u\left(v^{-1}(\mu)+2 \bar{\rho}\right) \mid u \in U\right\} \cap P_{+} .
$$

But $\mu+2 \bar{\rho} \in P_{+}$so we have $\left\{u\left(v^{-1}(\mu)+2 \bar{\rho}\right) \mid u \in U\right\} \cap P_{+}=\{\mu+2 \bar{\rho}\}$. Hence, there exists $u \in U$ such that $u\left(v^{-1}(\mu)+2 \bar{\rho}\right)=\mu+2 \bar{\rho}$. We have

$$
\begin{aligned}
& \mu+2 \bar{\rho}=u\left(v^{-1}(\mu)+2 \bar{\rho}\right) \\
& \text { 步 } \\
& u^{-1}(\mu+2 \bar{\rho})=v^{-1}(\mu)+2 \bar{\rho} \\
& \Uparrow \\
& v u^{-1}(\mu+2 \bar{\rho})=\mu+2 v(\bar{\rho}) \\
& \Uparrow \\
& v u^{-1}(\mu+2 \bar{\rho})-(\mu+2 \bar{\rho})=2(v(\bar{\rho})-\bar{\rho}) .
\end{aligned}
$$

Since $\mu+2 \bar{\rho} \in P_{+}$, we have $v u^{-1}(\mu+2 \bar{\rho})-(\mu+2 \bar{\rho}) \leq 0$. Hence $v(\bar{\rho}) \leq \bar{\rho}$. By Lemma 3.3, this implies that $v(\bar{\rho})=\bar{\rho}$. Finally by Lemma 3.2 , we have $v\left(\bar{R}_{+}\right)=\bar{R}_{+}$.

Remark 6.5. We will see in the next section (Remark 7.2) that we can have $\mu$ and $\nu$ in the same $W$-orbit, $\mu+2 \bar{\rho}$ and $\nu+2 \bar{\rho}$ in the same $W$-orbit but $H_{\mu} \neq H_{\nu}$. So the hypothesis $\mu+2 \bar{\rho} \in P_{+}$is crucial in the above proposition.

\section{The Classical Lie algebras}

7.1. Proof of the conjecture for $\mathfrak{g l}_{n}$. We now prove our conjecture in type $A$. We shall work in fact with $\mathfrak{g l}_{n}$ rather than $\mathfrak{s l}_{n}$. The main tool is a duality result between the branching coefficients $m_{\mu}^{\lambda}$ and some generalized Littlewood Richardson coefficients together with the main result of [6]. Each partition $\lambda=\left(\lambda_{1} \geq \cdots \geq \lambda_{d} \geq 0\right)$ can be regarded as a dominant weight of $\mathfrak{g l}_{n}$ by adding $n-d$ coordinates equal to 0 . We will use this convention in this section. For any partition $\mu=$ $\left(\mu_{1} \geq \cdots \geq \mu_{d}\right)$, we have in fact

$$
s_{\mu}=\sum_{\lambda=\left(\lambda_{1} \geq \cdots \geq \lambda_{d} \geq 0\right)} K_{\lambda, \mu}^{-1} h_{\lambda}
$$

that is, the coefficients appearing in the expansion of $s_{\mu}$ on the $h$-basis are inverse Kostka numbers indexed by pairs $(\lambda, \mu)$ of partitions with at most $d$ nonzero parts. When $\mathfrak{g}=\mathfrak{g l}_{n}$, the $h$-functions have also an additional property (which does not hold for the other root systems). Consider $\beta=$ $\left(\beta_{1}, \ldots, \beta_{n}\right) \in \mathbb{Z}_{\geq 0}^{n}$, then $h_{\beta}=h_{\beta_{1}} \times \cdots \times h_{\beta_{n}}$.

Recall that the dominant weights of $\mathfrak{g l}_{n}$ can be regarded as non increasing sequences of integers (possibly negative) with length $n$. We will realise $\overline{\mathfrak{g}}=\mathfrak{g l}_{m_{1}} \oplus \cdots \oplus \mathfrak{g l}_{m_{r}}$ as the subalgebra of $\mathfrak{g l}_{m}$ of block matrices with block sizes $m_{1}, \ldots, m_{r}$. Now consider $\mu \in \bar{P}$ such that $\mu=\mu^{(1)}+\cdots+\mu^{(r)}$ where $\mu^{(k)} \in P_{+}^{(k)}$ as in $\S 4.2$. Then each $\mu^{(k)}$ is a non increasing sequence of integers of length $m_{k}$. We will assume temporary that the coordinates of $\mu$ are nonnegative so that each $\mu^{(k)}$ is a partition with $m_{k}$ parts. We then have according to (2)

$$
H_{\mu}=\sum_{\lambda^{(1)} \in P_{+}^{(1)}} \cdots \sum_{\lambda^{(r)} \in P_{+}^{(r)}} K_{\lambda^{(1)}, \mu^{(1)}}^{-1} \cdots K_{\lambda^{(r)}, \mu^{(r)}}^{-1} h_{\lambda^{(1)}+\cdots+\lambda^{(r)}}
$$


where each $\lambda^{(k)}$ is a partition. In particular, we have $h_{\lambda^{(1)}+\cdots+\lambda^{(r)}}=h_{\lambda^{(1)}} \times \cdots \times h_{\lambda^{(r)}}$ which yields

$$
H_{\mu}=\prod_{i=1}^{k}\left(\sum_{\lambda^{(k)} \in P_{+}^{(k)}} K_{\lambda^{(k)}, \mu^{(k)}}^{-1} h_{\lambda^{(k)}}\right) .
$$

Finally by using (3), we obtain

$$
H_{\mu}=\prod_{i=1}^{k} s_{\mu^{(k)}} .
$$

We can now prove our conjecture for induced representations of $\mathfrak{g l}_{n}$

Proposition 7.1. Let $\mu$ and $\nu$ be dominant weights of $\overline{\mathfrak{g}}$. Assume $H_{\mu}=H_{\nu}$. Then, there exists a permuation $\sigma$ of $\{1, \ldots, n\}$ such that $\sigma\left(\bar{R}_{+}\right)=\bar{R}_{+}$.

Proof. By Theorem 2.2, we have $m_{\mu}^{\lambda}=\sum_{\sigma \in S_{n}} \varepsilon(\sigma) \overline{\mathcal{P}}(\sigma(\lambda+\rho)-\mu-\rho)$. Set $\delta=(1, \ldots, 1) \in \mathbb{Z}^{n}$. Since $\delta$ is fixed by $S_{n}$, we have for any nonnegative integer $a, m_{\lambda+a \delta}^{\mu+a \delta}=m_{\lambda}^{\mu}$. Observe also that $P_{+}$ is invariant by translation by $\delta$. Therefore

$$
H_{\mu+\delta a}=\sum_{\nu \in P_{+}} m_{\nu}^{\mu+a \delta} s_{\nu}=\sum_{\lambda \in P_{+}} m_{\lambda+a \delta}^{\mu+a \delta} s_{\lambda+a \delta}=\sum_{\lambda \in P_{+}} m_{\lambda}^{\mu} s_{\lambda+a \delta}
$$

by setting $\nu=\lambda+a \delta$ in the leftmost sum. So $H_{\mu}=H_{\nu}$ if and only if $H_{\mu+a \delta}=H_{\nu+a \delta}$. We can now choose $a$ sufficiently large so that $\mu \in \mathbb{Z}_{>0}^{n}$ and $\nu \in \mathbb{Z}_{>0}^{n}$. Decompose $\mu=\mu^{(1)}+\cdots+\mu^{(r)}$ and $\nu=\nu^{(1)}+\cdots+\nu^{(r)}$ as in $\S 4.2$. For any $k=1, \ldots, r$, set $\delta_{k}=(1, \ldots, 1) \in \mathbb{Z}^{m_{k}}$. The similar decompositions of $\mu+a \delta$ and $\nu+a \delta$ verify $(\mu+a \delta)^{(k)}=\mu^{(k)}+a \delta^{(k)}$ and $(\nu+a \delta)^{(k)}=\nu^{(k)}+a \delta^{(k)}$ for any $k=1, \ldots, r$. We thus obtain

$$
\prod_{i=1}^{k} s_{\mu^{(k)}+a \delta^{(k)}}=\prod_{i=1}^{k} s_{\nu^{(k)}+a \delta^{(k)}} .
$$

Now by the main result of [6], since the partitions $\mu^{(k)}+a \delta^{(k)}$ and $\nu^{(k)}+a \delta^{(k)}$ appearing above have positive parts, we know that the set of partitions $\left\{\mu^{(k)}+a \delta^{(k)}, k=1, \ldots r\right\}$ and $\left\{\nu^{(k)}+a \delta^{(k)}, k=\right.$ $1, \ldots r\}$ coincide. There thus exists a permuation $\tau \in S_{r}$ such that $\mu^{(k)}+a \delta^{(k)}=\nu^{(\tau(k))}+a \delta^{(\tau(k))}$. The permuation $\tau$ preserves the lengths of the partitions so $m_{k}=m_{\tau(k)}$ and $\delta^{(k)}=\delta^{(\tau(k))}$ for any $k=1, \ldots, r$. We obtain $\mu^{(k)}=\nu^{(\tau(k))}$. For any $k=1, \ldots, r$, set $I_{k}=\left\{m_{k-1}+1, \ldots, m_{k}\right\}$ (with $\left.m_{0}=0\right\}$. Then $I_{k}$ and $I_{\tau(k)}$ have the same cardinality because $m_{k}=m_{\tau(k)}$. Let $\sigma \in S_{n}$ be such that $\sigma\left(m_{k-1}+j\right)=m_{\tau(k)-1}+j$ for any $j \in\{1, \ldots, k\}$ and any $k \in\{1, \ldots, r\}$. Then $\sigma$ is a Dynkin diagram automorphism of $\overline{\mathfrak{g}}$. We have $\sigma(\mu)=\nu$ and $\sigma\left(\bar{R}_{+}\right)=\bar{R}_{+}$as desired.

Remark 7.2. Observe that we can have $\mu$ and $\nu$ in the same $W$-orbit, $\mu+2 \bar{\rho}$ and $\nu+2 \bar{\rho}$ in the same $W$-orbit but $H_{\mu} \neq H_{\nu}$. Consider for example $\overline{\mathfrak{g}}=\mathfrak{g l}_{4} \oplus \mathfrak{g l}_{2}$ in $\mathfrak{g l}_{6}$ and $\mu=(5,2,2,1 \mid 4,3)$ and $\nu=(5,4,3,1 \mid 2,2)$. We have $2 \bar{\rho}=(3,1,-1,-3 \mid 1,-1)$ so $\mu+2 \bar{\rho}=(8,3,1,-2 \mid 5,2)$ and $\nu+2 \bar{\rho}=(8,5,2,-2 \mid 3,1)$ belong to the same $W$-orbit. By the previous proposition, we have $H_{\mu} \neq H_{\nu}$. We cannot apply Proposition 6.4 since neither $\mu+2 \bar{\rho}$ or $\nu+2 \bar{\rho}$ belongs to $P_{+}$. 
7.2. Polarisation. Assume $\mathfrak{g}=\mathfrak{s o}_{2 n+1}, \mathfrak{s p}_{2 n}$ or $\mathfrak{s o}_{2 n}$ and $\overline{\mathfrak{g}}=\mathfrak{g l}_{n}$. Each dominant weight $\mu \in \bar{P}_{+}$ defines a pair of partitions $\left(\mu_{+}, \mu_{-}\right)$of length $\leq n$ obtained by ordering decreasingly the positive and negative coordinates of $\mu$, respectively. Recall also that to each partition $\lambda$ of length $\leq n$ corresponds a dominant weight of $P_{+}$. The branching coefficients $m_{\mu}^{\lambda}$ were obtained by Littelwood (see [5]). They can be expressed in terms of the Littelwood-Richardson coefficients as follows:

$$
m_{\mu}^{\lambda}= \begin{cases}\sum_{\gamma, \delta} c_{\mu_{+}, \mu_{-}}^{\gamma} c_{\gamma, \delta}^{\lambda} & \text { for } \mathfrak{g}=\mathfrak{s o}_{2 n+1}, \\ \sum_{\gamma, \delta} c_{\mu_{+}, \mu_{-}}^{\gamma} c_{\gamma, 2 \delta}^{\lambda} & \text { for } \mathfrak{g}=\mathfrak{s p}_{2 n}, \\ \sum_{\gamma, \delta} c_{\mu_{+}, \mu_{-}}^{\gamma} c_{\gamma,(2 \delta)^{*}}^{\lambda} & \text { for } \mathfrak{g}=\mathfrak{s o}_{2 n},\end{cases}
$$

where $\gamma$ and $\delta$ runs over the set of partitions with length $\leq n$ and $(2 \delta)^{*}$ is the conjugate partition of $2 \delta$.

Proposition 7.3. Conjecture 4.5 is true for $\mathfrak{g}=\mathfrak{s o}_{2 n+1}, \mathfrak{s p}_{2 n}$ or $\mathfrak{s o}_{2 n}$ and $\overline{\mathfrak{g}}=\mathfrak{g l}_{n}$.

Proof. Consider $\mu$ and $\nu$ in $\bar{P}_{+}$such that $H_{\mu}=H_{\nu}$. We have $m_{\mu}^{\lambda}=m_{\nu}^{\lambda}$ for any $\lambda \in P_{+}$. For any partition $\lambda$, write $|\lambda|$ the size of $\lambda$, that is the sum of its parts. Observe first that $m_{\mu}^{\lambda}=0$ when $|\lambda|<\left|\mu_{+}\right|+\left|\mu_{-}\right|$. Also, when $|\lambda|=\left|\mu_{+}\right|+\left|\mu_{-}\right|$in the above branching coefficients, we get $\delta=\emptyset, \gamma=\lambda$ and $m_{\mu}^{\lambda}=c_{\mu_{+}, \mu_{-}}^{\lambda}$ for $\mathfrak{g}=\mathfrak{s o}_{2 n+1}, \mathfrak{s p}_{2 n}$ or $\mathfrak{s o}_{2 n}$.

Assume $\left|\mu_{+}\right|+\left|\mu_{-}\right|<\left|\nu_{+}\right|+\left|\nu_{-}\right|$. Then for $\lambda=\mu_{+}+\mu_{-}$, we have $m_{\mu}^{\lambda}=c_{\mu_{+}, \mu_{-}}^{\lambda}=1$ whereas $m_{\nu}^{\lambda}=0$ since $|\lambda|=\left|\mu_{+}\right|+\left|\mu_{-}\right|<\left|\nu_{+}\right|+\left|\nu_{-}\right|$. So we obtain a contradiction. Similarly, we cannot have $\left|\mu_{+}\right|+\left|\mu_{-}\right|>\left|\nu_{+}\right|+\left|\nu_{-}\right|$. Therefore $\left|\mu_{+}\right|+\left|\mu_{-}\right|=\left|\nu_{+}\right|+\left|\nu_{-}\right|$. Then for any $\lambda$ such that $|\lambda|=\left|\mu_{+}\right|+\left|\mu_{-}\right|=\left|\nu_{+}\right|+\left|\nu_{-}\right|$, we have $c_{\mu_{+}, \mu_{-}}^{\lambda}=c_{\nu_{+}, \nu_{-}}^{\lambda}$. By the main result of [6], we obtain the equality of sets $\left\{\mu_{+}, \mu_{-}\right\}=\left\{\nu_{+}, \nu_{-}\right\}$. When $\mu_{+}=\nu_{+}$and $\mu_{-}=\nu_{-}$, we have $\mu=\nu$ and the conjecture holds. When $\mu_{+}=\nu_{-}$and $\mu_{-}=\nu_{+}$, we have $\mu=-\bar{w}_{0} \nu$ where $\bar{w}_{0}$ is the longest element of $\bar{W}$ that is, the permutation of $\{1, \ldots, n\}$ such that $w_{0}(k)=n-k+1$. Since $-\bar{w}_{0} \in W$ and $-w_{0}\left(\bar{R}_{+}\right)=\bar{R}_{+}$we are done.

We now summarize our results.

Theorem 7.4. Consider $\mu, \nu \in \bar{P}_{+}$.

(1) When $\mu$ and $\nu$ are conjugate under the action of a Dynkin diagram automorphism of $\overline{\mathfrak{g}}$ lying in $W$, we have $H_{\mu}=H_{\nu}$.

(2) Conversely, if we assume $H_{\mu}=H_{\nu}$, then $\mu$ and $\nu$ are conjugate under the action of a Dynkin diagram automorphism lying in $W$ when one of the following hypotheses is satisfied

- $\mu$ and $\nu$ belong to the same Weyl chamber of $\mathfrak{g}$ (in which case $\mu=\nu$ ),

- $\mu$ and $\nu$ are far enough of the walls of the Weyl chamber where they appear (each set $E_{\mu}$ or $E_{\nu}$ is entirely contained in a Weyl chamber),

- $\mu+2 \bar{\rho}$ or $\nu+2 \bar{\rho}$ belongs to $P_{+}$,

- $\mathfrak{g}=\mathfrak{g l}_{n}$,

- $\mathfrak{g}=\mathfrak{s o}_{2 n+1}, \mathfrak{s p}_{2 n}$ or $\mathfrak{s o}_{2 n}$ and $\overline{\mathfrak{g}}=\mathfrak{g l}_{n}$.

\section{REFERENCES}

[1] N. Bourbaki: Groupes et algèbres de Lie, Chapitres 4,5,6, Springer (2007).

[2] Duan, On the inverse Kostka matrix, Journal of Combinatorial Theory Series A, 103, 363-376, (2003).

[3] G. Goodman, N. R Wallach, Representation theory and invariants of the classical groups, Cambridge University Press.

[4] J. E. Humphreys. Reflection groups and Coxeter groups. Cambridge studies in advance mathematics 29, Cambridge University Press, (1990). 
[5] D.E. Littlewood, The theory of group characters and matrix representations of groups, Oxford University Press, second edition (1958).

[6] C.S. Rajan, Unique decomposition of tensor products of irreducible representations of simple algebraic groups Ann. of Math. 160, no. 2, 683-704. (2004).

[7] R. Venkatesh and Sankaran Viswanath, Unique factorization of tensor products for Kac-Moody algebras, Adv. in Math. 231, no. 6, 3162-3171(2012).

[8] M. Wildon, Character values and decomposition matrices of symmetric groups, J. Algebra 319, 3382-3397 (2008). 